\title{
Status of strains that contravene Rules 27(3) and 30 of the Bacteriological Code. Request for an Opinion
}

\author{
Correspondence \\ J. P. Euzéby \\ j.euzeby@envt.fr
}

\author{
J. P. Euzéby ${ }^{1}$ and B. J. Tindall ${ }^{2}$ \\ 'Société de Bactériologie Systématique et Vétérinaire (SBSV) and École Nationale Vétérinaire \\ de Toulouse (ENVT), 23 chemin des Capelles, 31076 Toulouse cedex 3, France \\ ${ }^{2}$ DSMZ-Deutsche Sammlung von Mikroorganismen und Zellkulturen GmbH, Mascheroder Weg \\ 1b, D-38124 Braunschweig, Germany
}

In the period from January 2001, at least 207 new names proposed in articles in the International Journal of Systematic and Evolutionary Microbiology or cited in Validation Lists are not in accordance with Rules 27(3) and 30 of the Bacteriological Code. The purpose of the present Request for an Opinion is to clarify the status of the names listed and to provide a solution whereby they may be considered to be validly published.
The Rules of the Bacteriological Code governing the deposit of type strains were modified on 14 August 1999 by the Judicial Commission during its meetings in Sydney, Australia (De Vos \& Trüper, 2000). Thereafter, the International Committee on Systematic Bacteriology (ICSB) (now International Committee on Systematics of Prokaryotes, ICSP) also voted unanimously in favour of these changes at the Sydney meeting (Labeda, 2000).

The revised Rule 27 states:

'The name of a new taxon, or a new combination for an existing taxon, is not validly published unless the following criteria are met.

(3) The type of the taxon must be designated. In the case of species or subspecies the culture collection numbers of at least two publicly accessible service collections in different countries where a subculture of the type strain has been deposited must be indicated.'

The revised Rule 30 states: 'For the name of species to be validly published it must conform with the following conditions.

(1) It must be published in conformity with the Rules 27 and $28 \mathrm{~b}$.

(2) It must be published as a binary combination consisting of a genus name followed by a single species epithet (see Rule 12a).

(3a) Up to the publication of the acceptance of these Minutes by the IJSEM, before publication of the name of a

Published online ahead of print on 21 November 2003 as DOI 10.1099/ijs.0.03022-0. new species, a culture of the type strain (or, if the species is non-cultivable, type material, a photograph or an illustration) should be deposited in at least one of the permanently established culture collections from which it would be readily available. The designation allotted to the strain by the culture collections should be quoted in the published description.

(3b) As of the publication date of the acceptance of these Minutes by the IJSEM, the description must include the designation of a type strain, and a viable culture of that strain must be deposited in at least two publicly accessible service collections in different countries from which subcultures must be available. The designations allotted to the strain by the culture collections should be quoted in the published description.

(4) Patent strains may serve as type strains and must also be deposited as in (3a) or (3b). Patent strains must be clearly identified as such at the time of publication. If the patent is not yet awarded or not laid open, the publication of a name and the description has to be deferred until the patent is awarded or subcultures become available.'

Minutes of the meetings of the Judicial Commission and of the ICSP were published in the November 2000 issue of the IJSEM (De Vos \& Trüper, 2000; Labeda, 2000). As the publication date of this issue was 14 December 2000, for all practical purposes the revised Rules 27(3) and 30 apply from 1 January 2001. However, it should be noted that members of the Judicial Commission or ICSP serving on the Editorial Board of IJSEM, who were at the Sydney meeting, should have been aware of these forthcoming changes, and that these requirements were published on the IJSEM web site under the section 'Guidelines for Authors' well in advance of the publication of the Minutes. 
Table 1. New species, new subspecies or new combinations where type strains are not deposited in any recognized service collection or deposited in a single recognized culture collection or deposited in at least two different recognized culture collections in the same country

To the authors' knowledge (as of 9 October 2003), a deposit in another recognized culture collection in a different country is not documented.

\begin{tabular}{|c|c|}
\hline Name of taxon and author(s) & Type strain(s) cited in effective publication \\
\hline Acetobacter cibinongensis Lisdiyanti et al. 2002 & $4 \mathrm{H}-1=\mathrm{IFO}($ now NBRC) $16605=\mathrm{JCM} 11196$ \\
\hline Acetobacter orientalis Lisdiyanti et al. 2002 & $21 \mathrm{~F}-2=\mathrm{IFO}($ now NBRC) $16606=\mathrm{JCM} 11195$ \\
\hline Acetobacter syzygii Lisdiyanti et al. 2002 & 9H-2 = IFO (now NBRC) $16604=J C M 11197$ \\
\hline Acetobacterium tundrae Simankova et al. 2001 & $Z-4493=$ DSM 9173 \\
\hline Actinoplanes capillaceus Matsumoto et al. 2001 & K95-5561 =JCM $10268=$ IFO (now NBRC) 16408 \\
\hline Actinoplanes friuliensis Aretz et al. 2001 & HAG $010964=$ DSM 7358 \\
\hline Aeromonas culicicola Pidiyar et al. 2002 & MTCC $3249=$ NCIM 5147 \\
\hline Alicyclobacillus acidocaldarius subsp. rittmannii Nicolaus et al. 2002 & MR1 = DSM 11297 \\
\hline Alkalispirillum mobile Rijkenberg et al. 2002 & SL-1 = DSM 12769 \\
\hline Anaeromyxobacter dehalogenans Sanford et al. 2002 & $2 \mathrm{CP}-1=\mathrm{ATCC}$ BAA-258 \\
\hline Asaia siamensis Katsura et al. 2001 & $\begin{array}{l}\text { S60-1= JCM } 10715=\text { IFO (now NBRC) } 16457= \\
\text { NRIC } 0323\end{array}$ \\
\hline Azoarcus buckelii Mechichi et al. 2002 & $\mathrm{U} 120=\mathrm{DSM} 14744$ \\
\hline Azospirillum doebereinerae Eckert et al. 2001 & GSF71 = DSM 13131 \\
\hline Bacillus thermantarcticus corrig. Nicolaus et al. 2002 & M1 = DSM 9572 \\
\hline Borrelia sinica Masuzawa et al. 2001 & $\mathrm{CMN3}=\mathrm{JCM} 10505$ \\
\hline Bradyrhizobium yuanmingense Yao et al. 2002 & CCBAU $10071=$ CFNEB 101 \\
\hline Chlorobaculum limnaeum Imhoff 2003 & DSM 1677 \\
\hline Chlorobaculum parvum Imhoff 2003 & DSM 263 \\
\hline Chlorobaculum thiosulfatiphilum Imhoff 2003 & DSM 249 \\
\hline Chlorobium clathratiforme (Szafer 1911) Imhoff 2003 & BU1 = DSM 5477 \\
\hline Chlorobium luteolum (Schmidle 1901) Imhoff 2003 & DSM 273 \\
\hline Clostridium phytofermentans Warnick et al. 2002 & ISDg $=$ ATCC 700394 \\
\hline Comamonas koreensis Chang et al. 2002 & $\mathrm{YH} 12=\mathrm{KCTC} 12005=\mathrm{IMSNU} 11158$ \\
\hline Dehalospirillum multivorans Scholz-Muramatsu et al. 2002 & DSM 12446 \\
\hline Desulfobulbus mediterraneus Sass et al. 2002 & $86 \mathrm{FS} 1=\mathrm{DSM} 13871$ \\
\hline Desulfomicrobium orale Langendijk et al. 2001 & NY678=DSM 12838 \\
\hline Desulfomonile limimaris Sun et al. 2001 & DCB-M = ATCC 700979 \\
\hline Desulfovibrio dechloracetivorans Sun et al. 2001 & $\mathrm{SF} 3=\mathrm{ATCC} 700912$ \\
\hline Desulfovibrio mexicanus Hernandez-Eugenio et al. 2001 & Lupl=DSM 13116 \\
\hline Desulfovibrio vietnamensis Dang et al. 2002 & G3 $100=$ DSM 10520 \\
\hline Dethiosulfovibrio acidaminovorans Surkov et al. 2001 & SR $15=$ DSM 12590 \\
\hline Dethiosulfovibrio marinus Surkov et al. 2001 & WS $100=$ DSM 12537 \\
\hline Dethiosulfovibrio russensis Surkov et al. 2001 & SR $12=$ DSM 12538 \\
\hline Ensifer kummerowiae (Wei et al. 2002) Young 2003 & AS $1.3046=$ CCBAU 71714 \\
\hline Enterobacter cowanii Inoue et al. 2001 & $888-76=\mathrm{JCM} 10956$ \\
\hline Geoglobus ahangari Kashefi et al. 2002 & $234=$ ATCC BAA -425 \\
\hline Hahella chejuensis Lee et al. 2001 & 96CJ10356=KCTC 2396=IMSNU 11157 \\
\hline Haloferax alexandrinus Asker and Ohta 2002 & $\mathrm{TM}=\mathrm{JCM} 10717=\mathrm{IFO}($ now NBRC) 16590 \\
\hline Halorhodospira neutriphila Hirschler-Réa et al. 2003 & SG $3301=$ DSM 15116 \\
\hline Helicobacter typhlonius Franklin et al. 2002 & MIT $97-6810=$ ATCC BAA-367 \\
\hline Heliobacterium sulfidophilum Bryantseva et al. 2001 & $\mathrm{BR} 4=\mathrm{UNIQEM} 113$ \\
\hline Heliorestis baculata Bryantseva et al. 2001 & OS-H1=DSM 13446 \\
\hline Herbaspirillum frisingense Kirchhof et al. 2001 & GSF30=DSM 13128 \\
\hline Hydrogenobacter subterraneus Takai et al. 2001 & $\mathrm{HGP} 1=\mathrm{IFO}($ now NBRC) $16485=\mathrm{JCM} 10560$ \\
\hline Hydrogenophaga intermedia Contzen et al. 2001 & $\mathrm{~S} 1=\mathrm{DSM} 5680$ \\
\hline Ketogulonicigenium vulgare corrig. Urbance et al. 2001 & DSM 4025 \\
\hline Lactobacillus ferintoshensis Simpson et al. 2002 & $\mathrm{R} 7-84=\mathrm{CIP} 106749$ \\
\hline Leptotrichia trevisanii Tee et al. 2002 & 'Wee Tee' $1999=$ ATCC 700907 \\
\hline Leuconostoc gasicomitatum Björkroth et al. 2001 & TB $1-10=\mathrm{LMG} 18811$ \\
\hline Marinospirillum alkaliphilum Zhang et al. 2002 & $\mathrm{Z} 4=\mathrm{CGMCC}$ AS 1.2746 \\
\hline
\end{tabular}


Table 1. cont.

\begin{tabular}{|c|c|}
\hline Name of taxon and author(s) & Type strain(s) cited in effective publication \\
\hline Methanocaldococcus fervens (Jeanthon et al. 1999) Whitman 2002 & AG86 = DSM 4213 \\
\hline Methanocaldococcus infernus (Jeanthon et al. 1998) Whitman 2002 & $\mathrm{ME}=\mathrm{DSM} 11812$ \\
\hline Methylomicrobium buryatense Kaluzhnaya et al. 2001 & $5 \mathrm{~B}=\mathrm{VKM} \mathrm{B}-2245$ \\
\hline Methylovorus mays Doronina et al. 2001 & $\mathrm{BV}=\mathrm{VKM} \mathrm{B}-2221$ \\
\hline Microcystis aeruginosa Otsuka et al. 2001 (illegitimate name) & NIES843 = IAM M-247 \\
\hline Mycobacterium heckeshornense Roth et al. 2001 & $\mathrm{~S} 369=\mathrm{DSM} 44428$ \\
\hline Mycoplasma agassizii Brown et al. 2001 & PS6 $=$ ATCC $700616^{*}$ \\
\hline Mycoplasma alligatoris Brown et al. 2001 & A21JP2=ATCC 700619 \\
\hline Mycoplasma microti Brown et al. 2001 & IL371 = ATCC 700935 \\
\hline Nautilia lithotrophica Miroshnichenko et al. 2002 & $525=$ DSM 13520 \\
\hline Neochlamydia hartmannellae Horn et al. 2001 & $\mathrm{~A}_{1} \mathrm{Hsp}=\mathrm{ATCC} 50802$ \\
\hline Neorickettsia risticii (Holland et al. 1985) Dumler et al. 2001 & Illinois $=$ HRC-IL $=$ ATCC VR-986 (deaccessioned $)$ \\
\hline Neorickettsia sennetsu (Misao and Kobayashi 1956) Dumler et al. 2001 & Miyayama $=$ ATCC VR-367 \\
\hline Nitrobacter hamburgensis Bock et al. 2001 & $\mathrm{X} 14=\mathrm{DSM} 10229$ \\
\hline Nitrobacter vulgaris Bock et al. 2001 & $\mathrm{Z}=\mathrm{DSM} 10236$ \\
\hline Nitrosomonas aestuarii Koops et al. 2001 & $\mathrm{Nm} 36 \dagger$ \\
\hline Nitrosomonas communis Koops et al. 2001 & $\mathrm{Nm} 2 \dagger$ \\
\hline Nitrosomonas eutropha Koops et al. 2001 & $\mathrm{Nm} 57 \dagger$ \\
\hline Nitrosomonas halophila Koops et al. 2001 & $\mathrm{Nm} 1 \dagger$ \\
\hline Nitrosomonas marina Koops et al. 2001 & $\mathrm{Nm} 22 \dagger$ \\
\hline Nitrosomonas nitrosa Koops et al. 2001 & $\mathrm{Nm} 90 \dagger$ \\
\hline Nitrosomonas oligotropha Koops et al. 2001 & $\mathrm{Nm} 45 \dagger$ \\
\hline Nitrosomonas ureae Koops et al. 2001 & $\mathrm{Nm} 10 \dagger$ \\
\hline Nitrospira moscoviensis Ehrich et al. 2001 & NSP M-1=DSM 10035 \\
\hline Opitutus terrae Chin et al. 2001 & PB90-1 = DSM 11246 \\
\hline Paenibacillus granivorans Van der Maarel et al. 2001 & $\mathrm{~A} 30=\mathrm{CBS} 229.89$ \\
\hline Planococcus alkanoclasticus Engelhardt et al. 2001 & MAE2 = NCIMB 13489 \\
\hline Prochlorococcus marinus Chisholm et al. 2001 & CCMP-1375 \\
\hline Prochlorococcus marinus subsp. marinus Chisholm et al. 2001 & CCMP-1375 \\
\hline Propionispora vibrioides Biebl et al. 2001 & $\mathrm{FKBS} 1=\mathrm{DSM} 13305$ \\
\hline Prosthecochloris vibrioformis (Pelsh 1936) Imhoff 2003 & DSM 260 \\
\hline Pseudomonas cedrina corrig. Dabboussi et al. 2002 & CFML 96-198=CIP 105541 \\
\hline Pseudomonas cremoricolorata Uchino et al. 2002 & $\begin{array}{l}\text { CC- } 8=\text { NRIC } 0181=\text { IFO }(\text { now NBRC) } 16634= \\
\text { JCM } 11246\end{array}$ \\
\hline Pseudomonas orientalis Dabboussi et al. 2002 & CFML 96-170=CIP 105540 \\
\hline Pseudomonas parafulva Uchino et al. 2002 & $\begin{array}{l}\mathrm{CB}-1=\mathrm{AJ} 2129=\mathrm{IFO}(\text { now NBRC) } 16636=\mathrm{JCM} \\
11244=\mathrm{NRIC} 0501\end{array}$ \\
\hline Pseudomonas psychrophila Yumoto et al. 2002 & $\mathrm{E}-3=\mathrm{JCM} 10889$ \\
\hline Rhizobium indigoferae Wei et al. 2002 & AS $1.3045=$ CCBAU 71042 \\
\hline Rhodobaca bogoriensis Milford et al. 2001 & $\mathrm{LBB} 1=\mathrm{ATCC} 700920$ \\
\hline Rhodoferax antarcticus Madigan et al. 2001 & ANT.BR $=$ ATCC 700587 \\
\hline Rickettsia felis Bouyer et al. 2001 & Strain Ctenocephalides felis-LSU \\
\hline Rickettsia felis Bouyer et al. 2001 emend. La Scola et al. 2002 & CNCM I-2363 \\
\hline Roseospirillum parvum Glaeser and Overmann 2001 & $930 \mathrm{I}=\mathrm{DSM} 12498$ \\
\hline Selenihalanaerobacter shriftii Switzer Blum et al. 2001 & DSSe-1 = ATCC BAA-73 \\
\hline Sinorhizobium kummerowiae Wei et al. 2002 & AS $1.3046=$ CCBAU 71714 \\
\hline Sinorhizobium morelense Wang et al. 2002 & $\mathrm{Lc} 04=\mathrm{CFN}$ E1007 = LMG 21331 \\
\hline Sulfolobus tokodaii Suzuki et al. 2002 & $7=\mathrm{JCM} 10545$ \\
\hline Sulfurospirillum multivorans (Scholz-Muramatsu et al. 2002) Luijten et al. 2003 & DSM 12446 \\
\hline Syntrophus aciditrophicus Jackson et al. 2001 & $\mathrm{SB}=\mathrm{ATCC} 700169$ \\
\hline Thauera aminoaromatica Mechichi et al. 2002 & $\mathrm{~S} 2=\mathrm{DSM} 14742$ \\
\hline Thauera chlorobenzoica Song et al. 2001 & $3 \mathrm{CB}-1=$ ATCC 700723 \\
\hline Thauera phenylacetica Mechichi et al. 2002 & $\mathrm{~B} 4 \mathrm{P}=\mathrm{DSM} 14743$ \\
\hline Thermococcus sibiricus Miroshnichenko et al. 2001 & MM $739=$ DSM 12597 \\
\hline
\end{tabular}


Table 1. cont.

\begin{tabular}{|lc|}
\hline Name of taxon and author(s) & Type strain(s) cited in effective publication \\
\hline Thermoproteus uzoniensis Bonch-Osmolovskaya et al. 2001 & Z-605=DSM 5263 \\
Thioflavicoccus mobilis Imhoff and Pfennig 2001 & $8321=$ ATCC 700959 \\
Treponema parvum Wyss et al. 2001 & OMZ $833=$ ATCC 700770 \\
Trichlorobacter thiogenes De Wever et al. 2001 & K1 $=$ ATCC BAA-34 \\
Tropheryma whipplei La Scola et al. 2001 & CNCM I-2202\$ \\
\hline
\end{tabular}

*The type strain is also available from the Mollicutes Culture Collection (Curators Drs J. K. Davis and M. K. Davidson, University of Florida). $\dagger$ Strains deposited at the culture collection of the Institut für allgemeine Botanik, Mikrobiologische Abteilung, Universität Hamburg, Germany. $\ddagger$ The type strain is also available upon request from the authors.

§NCM numbers appear to be issued solely for patent purposes. In the cases listed, the status of these strains as patent strains is not documented, nor does evidence appear to have been presented that the relevant patent has either been issued or laid open. The type strains have been also deposited in the Collection de l'Unite des Rickettsies (WHO Collaborative Center for Rickettsial Reference and Research, Marseilles, France).

Unfortunately, some new species, new subspecies and new combinations, published in the IJSEM subsequent to 1 January 2001, are not in accordance with the revised Rules 27(3) and 30. These names are listed in this paper in four different tables. It should be emphasized that the Judicial Commission and the ICSP introduced the changes in these two Rules in order to counter the undesirable trend whereby unreasonable restrictions were placed on the strains or where access was being denied. It should be noted that some exceptions may be made, particularly in cases where only a limited number of collections have access to certain technical facilities (e.g. extreme barophiles or organisms of Risk Group 3). The deposit of strains in at least two different collections also ensures a system whereby type material is stored in at least two different global locations, ensuring the safe storage of this material.

Table 1 lists new species, new subspecies names and new combinations where (a) the type strain is not deposited in any recognized service collection; (b) the type strain is only known to have been deposited in a single culture collection; (c) the type strain is deposited in at least two different culture collections in the same country; or (d) in some cases it is unclear whether additional acronyms refer to other, unidentified, internationally recognized service culture collections.

The names included in this table provide the greatest problems, although we suggest that this Request for an Opinion be used as a mechanism by which this problem is made known and the authors of these names, or curators of service culture collections are asked to supply information on the additional deposit of these strains in at least a second internationally recognized service collection in a second country. In the event of such deposits being made known to the Judicial Commission, these names should be regarded as validly published. We also suggest that this be co-ordinated via the current List Editor.

Table 2 lists new species or new combinations where the effective publication only documents deposit in a single internationally recognized service culture collection, or in two culture collections in the same country. In view of the fact that we have been able to document the deposit of the type strain in other internationally recognized culture collections, we suggest that these names be treated as having being validly published at the time of their effective publication in the IJSEM.

Table 3 lists new subspecies or new combinations where the effective publication makes no reference to the deposit of the type strain in any internationally recognized service culture collection. In view of the fact that we have been able to document the deposit of the type strain in at least two internationally recognized service culture collections, in two different countries, we suggest that these names be treated as having being validly published at the time of their effective publication in the IJSEM.

Table 4 lists new species where the protologue in the effective publication makes reference to the deposit of the type strain in either only one internationally recognized service culture collection, or in two internationally recognized service culture collections in the same country. However, in view of the fact that the authors also document the deposit of the type strain in at least two internationally recognized service culture collections in two different collections (usually in the Abstract), we suggest that these names be treated as having being validly published at the time of their effective publication in the IJSEM.

We would also like to draw the international microbiological community to the importance of following the Rules on the deposit of type strains in internationally recognized collections. These organisms serve as irreplaceable viable documents of the microbial diversity on this planet and they are the 'cultural heritage' of modern prokaryotic systematics. The type strain system also gives us unique access to the genetic, biochemical and physiological properties of that material, which is impossible with the vast majority of type material available in botany and zoology. 


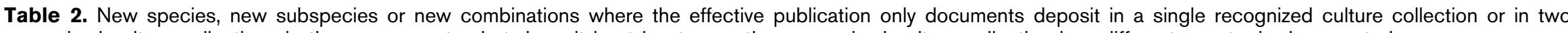
recognized culture collections in the same country, but deposit in at least one other recognized culture collection in a different country is documented

\begin{tabular}{|lcc}
\hline Name of taxon and author(s) & $\begin{array}{c}\text { Accession number cited in } \\
\text { effective publication }\end{array}$ \\
\hline
\end{tabular}

Actinocorallia aurantiaca (Lavrova and Preobrazhenskaya 1975) Zhang et al. 2001

Actinocorallia glomerata (Itoh et al. 1996) Zhang et al. 2001

Actinocorallia libanotica (Meyer 1981) Zhang et al. 2001

Actinocorallia longicatena (Itoh et al. 1996) Zhang et al. 2001

Actinomadura viridilutea (Agre and Guzeva 1975) Zhang et al. 2001

Anaerobranca gottschalkii Prowe and Antranikian 2001

Anaerococcus octavius (Murdoch et al. 1997) Ezaki et al. 2001

Anaerococcus prevotii (Foubert and Douglas 1948) Ezaki et al. 2001

Bacillus arseniciselenatis corrig. Switzer Blum et al. 2001

Bacillus selenitireducens Switzer Blum et al. 2001

Carboxydibrachium pacificum corrig. Sokolova et al. 2001

Cellulosimicrobium cellulans (Metcalf and Brown 1957) Schumann et al. 2001

Clostridium hungatei Monserrate et al. 2001

Clostridium saccharoperbutylacetonicum Keis et al. 2001

Comamonas denitrificans Gumaelius et al. 2001

Cryptosporangium aurantiacum (ex Ruan et al. 1976) Tamura and Hatano 2001

Desulfitobacterium chlororespirans Sanford et al. 2001

Desulfitobacterium metallireducens Finneran et al. 2002

Desulfobacula phenolica (Bak and Widdel 1988) Kuever et al. 2001

Desulfotignum balticum Kuever et al. 2001

Desulfotomaculum thermobenzoicum subsp. thermosyntrophicum Plugge et al. 2002

Dorea formicigenerans (Holdeman and Moore 1974) Taras et al. 2002

Gallicola barnesae (Schiefer-Ullrich and Andreesen 1986) Ezaki et al. 2001

Geobacillus kaustophilus (Priest et al. 1989) Nazina et al. 2001

\section{JCM 8201}

JCM 9376

IFO (now NBRC) 14095

JCM 9377

JCM 3398

DSM 13577

NCTC 9810

ATCC 9321

$\mathrm{E} 1 \mathrm{H}=\mathrm{ATCC} 700614$

ATCC 700615

DSM 12653

DSM 43879

ATCC 700212

ATCC 27021 (derived from ATCC

13564)

ATCC 700936

IFO (now NBRC) $13967=$ JCM 324

ATCC 700175

ATCC BAA-636

DSM 7044

DSM 14055

ATCC 27755

DSM 3244

ATCC 8005
DSM $43924=I F O$ (now NBRC) $15554=I F O$

(now NBRC) $16147=$ IMET $9577=$ KCTC 9554

DSM 44360 =IFO (now NBRC) 15960

ATCC $35576=$ DSM $43554=J C M \quad 3284=J C M$

$10696=$ NRRL B-16097 $=$ VKM Ac-939

CIP $105488=$ DSM $44361=$ IFO $($ now NBRC)

15961

ATCC $33925=$ CCRC $13638=$ DSM $44433=$

IFO (now NBRC) $14480=J C M 7346$

ATCC BAA-51

DSM 11663

CCUG $41932=$ CIP $105881=$ DSM $20548=J C M$

$6508=$ NCTC 11806

DSM 15340

DSM 15326

ATCC BAA-271

ATCC $12830=$ CFBP $4267=$ CIP $103404=$

HAMBI $95=$ IFO (now NBRC) $15516=J C M$ $9965=$ LMG $16221=$ NRRL B $-2768=$ VKM

Ac- 1412

DSM 14427

DSM $14923=$ NCIMB 12606

CCUG 44425

AS $4.1052=$ DSM $46144=$ KCC A- $0241=$ KCTC $9529=$ NCIMB $12649=$ NRRL B-16698

DSM 11544

DSM 15288

ATCC $43956=$ DSM 3384

ATCC BAA-19

ATCC BAA-281

DSM 3992

ATCC 49795

CCUG $28890=$ CIP $106929=$ DSM $7263=$ LMG $9819=$ NCCB $30003=$ NRRL NRS -81 
Table 2. cont.

\begin{tabular}{|c|c|c|}
\hline Name of taxon and author(s) & $\begin{array}{l}\text { Accession number cited in } \\
\text { effective publication }\end{array}$ & $\begin{array}{l}\text { Other accession number(s) under } \\
\text { which type strain is available }\end{array}$ \\
\hline Geobacillus stearothermophilus (Donk 1920) Nazina et al. 2001 & DSM 22 & $\begin{array}{l}\text { ATCC } 12980=\text { CCM } 2062=\text { CCRC } 11092= \\
\text { CCUG } 26241=\text { CIP } 66.23=\text { IAM } 11062= \\
\text { IFO }(\text { now NBRC) } 12550=\text { JCM } 2501= \\
\text { KCTC } 1665=\text { KCTC } 1752=\text { LMG } 6939= \\
\text { NCCB } 75019=\text { NCFB } 1768=\text { NCIMB } \\
8923=\text { NCTC } 10339=\text { NRRL B- } 1172= \\
\text { VKM B-510 }\end{array}$ \\
\hline Geobacillus thermodenitrificans (Manachini et al. 2000) Nazina et al. 2001 & DSM 465 & ATCC $29492=$ LMG 17532 \\
\hline Geobacillus thermoglucosidasius (Suzuki et al. 1984) Nazina et al. 2001 & DSM 2542 & $\begin{array}{l}\text { ATCC } 43742=\text { CCUG } 28887=\text { CIP } 106930= \\
\text { LMG } 7137=\text { NRRL B-14516 }\end{array}$ \\
\hline Geobacillus thermoleovorans (Zarilla and Perry 1988) Nazina et al. 2001 & DSM 5366 & ATCC $43513=$ LMG 9823 \\
\hline Grimontia hollisae (Hickman et al. 1982) Thompson et al. 2003 & LMG 17719 & $\begin{array}{l}\text { ATCC } 33564=\text { CCUG } 13625=\text { CDC } 0075-80= \\
\text { CIP } 101886=\text { DSM } 15132=J C M 1283= \\
\text { NCTC } 11640\end{array}$ \\
\hline Helicobacter aurati Patterson et al. 2002 & MIT $97-5075 c=$ ATCC BAA-1 & CCUG 47791 \\
\hline Helicobacter canadensis Fox et al. 2002 & $\begin{array}{l}\text { NLEP-16143=ATCC } 700968= \\
\text { MIT } 98-5491\end{array}$ & CCUG 47163 \\
\hline Heliobacterium undosum Bryantseva et al. 2001 & DSM 13378 & UNIQEM 114 \\
\hline Lamprocystis purpurea (Eichler and Pfennig 1989) Imhoff 2001 & DSM 4197 & ATCC 49210 \\
\hline Legionella drozanskii Adeleke et al. 2001 & ATCC 700990 & CCUG 43885 \\
\hline Legionella fallonii Adeleke et al. 2001 & ATCC 700992 & CCUG 43887 \\
\hline Legionella rowbothamii Adeleke et al. 2001 & ATCC 700991 & CCUG 43886 \\
\hline Lentzea violacea (Lee et al. 2000) Labeda et al. 2001 & IMSNU 50388 & JCM 10975 \\
\hline Marinibacillus marinus (Rüger and Richter 1979) Yoon et al. 2001 & DSM 1297 & $\begin{array}{l}\text { ATCC } 29841=\text { CCUG } 28884=\text { CIP } 103308= \\
\text { LMG } 6930=\text { NRRL B-14321 }\end{array}$ \\
\hline Marinobacterium jannaschii (Bowditch et al. 1984) Satomi et al. 2002 & ATCC 27135 & $\begin{array}{l}\text { CIP } 103444=\text { DSM } 6295=\text { IFO }(\text { now NBRC) } \\
15466=\text { LMG } 6239\end{array}$ \\
\hline Marinobacterium stanieri (Baumann et al. 1983) Satomi et al. 2002 & ATCC 27130 & $\begin{array}{l}\text { CCUG } 16021=\text { CIP } 106697=\text { DSM } 7027= \\
\text { LMG } 6847\end{array}$ \\
\hline Methanocaldococcus jannaschii (Jones et al. 1984) Whitman 2002 & DSM 2661 & $\begin{array}{l}\text { ATCC } 43067=\text { CIP } 104961=\text { JCM } 10045= \\
\text { OCM } 168\end{array}$ \\
\hline Methanocaldococcus vulcanius (Jeanthon et al. 1999) Whitman 2002 & DSM 12094 & ATCC 700851 \\
\hline Methanothermococcus thermolithotrophicus (Huber et al. 1984) Whitman 2002 & DSM 2095 & ATCC $35097=$ JCM $10549=$ OCM 138 \\
\hline Methanotorris igneus (Burggraf et al. 1990) Whitman 2002 & DSM 5666 & JCM 11834 \\
\hline Methylophilus leisingeri corrig. Doronina and Trotsenko 2001 & VKM B-2013 & DSM 6813 \\
\hline Mycobacterium bovis subsp. caprae (Aranaz et al. 1999) Niemann et al. 2002 & $\mathrm{spc}-1=\mathrm{gM}-1=\mathrm{CIP} 105776$ & ATCC BAA- 824 \\
\hline Nocardia cyriacigeorgica corrig. Yassin et al. 2001 & IMMIB D-1627= DSM 44484 & JCM 11763 \\
\hline Nocardia vinacea Kinoshita et al. 2002 & $\begin{array}{l}\text { JCM } 10988=\text { IFO (now NBRC) } \\
16497\end{array}$ & DSM 44638 \\
\hline
\end{tabular}


Table 2. cont.

\begin{tabular}{|c|c|c|}
\hline Name of taxon and author(s) & $\begin{array}{l}\text { Accession number cited in } \\
\text { effective publication }\end{array}$ & $\begin{array}{l}\text { Other accession number(s) under } \\
\text { which type strain is available }\end{array}$ \\
\hline $\begin{array}{l}\text { Nonomuraea roseoviolacea subsp. roseoviolacea (Nonomura and Ohara 1971) } \\
\text { Zhang et al. } 1998\end{array}$ & IFO (now NBRC) 14098 & $\begin{array}{l}\text { AS } 4.1072=\text { ATCC } 27297=\text { CBS } 260.72= \\
\text { CCM } 3491=\text { CCRC } 13406=\text { CIP } 106924 \\
=\text { DSM } 43144=\text { JCM } 3145=\text { KCTC } 9283 \\
=\text { NCIMB } 11117=\text { NRRL B- } 16127= \\
\text { VKM Ac- } 909\end{array}$ \\
\hline Oceanobacter kriegii (Bowditch et al. 1984) Satomi et al. 2002 & ATCC 27133 & $\begin{array}{l}\text { CIP } 103443=\text { DSM } 6294=\text { IFO }(\text { now NBRC) } \\
15467=\text { LMG } 6238\end{array}$ \\
\hline Olsenella profusa Dewhirst et al. 2001 & DSM 13989 & CCUG $45371=$ CIP 106885 \\
\hline Olsenella uli (Olsen et al. 1991) Dewhirst et al. 2001 & VPI D76D-27C = ATCC 49627 & CCUG $31166=$ DSM 7084=LMG 11480 \\
\hline Paracoccus kondratievae Doronina and Trotsenko 2001 & VKM B-2222 & NCIMB 13773 \\
\hline Peptoniphilus asaccharolyticus (Distaso 1912) Ezaki et al. 2001 & ATCC 14963 & $\begin{array}{l}\text { CCUG } 9988=\text { CIP } 74.17=\text { DSM } 20463= \\
\text { NCTC } 11461\end{array}$ \\
\hline Peptoniphilus harei (Murdoch et al. 1997) Ezaki et al. 2001 & DSM 10020 & CIP $105323=$ NCTC 13076 \\
\hline Peptoniphilus indolicus (Christiansen 1934) Ezaki et al. 2001 & ATCC 29427 & CCUG $17639=$ DSM $20464=$ NCTC 11088 \\
\hline Peptoniphilus ivorii (Murdoch et al. 1997) Ezaki et al. 2001 & DSM 10022 & CIP $105325=$ NCTC 13078 \\
\hline Pseudomonas brenneri Baïda et al. 2002 & CFML 97-391=CIP 106646 & DSM 15294 \\
\hline Pseudospirillum japonicum (Watanabe 1959) Satomi et al. 2002 & ATCC 19191 & $\begin{array}{l}\text { CIP } 103373=\text { DSM } 7165=\text { IFO }(\text { now NBRC) } \\
15446=\text { LMG } 10146\end{array}$ \\
\hline Sphingomonas aquatilis Lee et al. 2001 & KCCM $41067=$ KCTC 2881 & IFO (now NBRC) $16722=J C M 11455$ \\
\hline Sphingomonas cloacae Fujii et al. 2001 & IAM $14885=\mathrm{JCM} 10874$ & CIP $107076=$ DSM 14926 \\
\hline Sphingomonas koreensis Lee et al. 2001 & KCCM $41069=$ KCTC 2882 & IFO (now NBRC) $16723=J C M 11456$ \\
\hline Sphingomonas taejonensis Lee et al. 2001 & KCCM $41068=$ KCTC 2884 & IFO (now NBRC) $16724=J C M 11457$ \\
\hline Streptococcus gallolyticus subsp. pasteurianus (Poyart et al. 2002) Schlegel et al. 2003 & NEM $1202=$ CIP 107122 & DSM 15351 \\
\hline Streptococcus lutetiensis Poyart et al. 2002 & NEM 782=CIP 106849 & DSM 15350 \\
\hline Streptococcus pasteurianus Poyart et al. 2002 & NEM $1202=$ CIP 107122 & DSM 15351 \\
\hline Tenacibaculum maritimum (Wakabayashi et al. 1986) Suzuki et al. 2001 & NCIMB 2154 & $\begin{array}{l}\text { ATCC } 43398=\text { CIP } 103528=\text { CCUG } 35198= \\
\text { IFO (now NBRC) } 15946=\text { LMG } 11612\end{array}$ \\
\hline Tenacibaculum ovolyticum (Hansen et al. 1992) Suzuki et al. 2001 & NCIMB 13127 & $\begin{array}{l}\text { ATCC } 51887=\text { CCUG } 35199=\text { CIP } 106403= \\
\text { LMG } 13026\end{array}$ \\
\hline Terasakiella pusilla (Terasaki 1973) Satomi et al. 2002 & IFO (now NBRC) 13613 & $\begin{array}{l}\text { ATCC } 33338=\text { CIP } 103382=\text { DSM } 6293= \\
\text { LMG } 7372\end{array}$ \\
\hline Thermococcus acidaminovorans Dirmeier et al. 2001 & DSM 11906 & JCM 12186 \\
\hline Thermococcus waiotapuensis González et al. 2001 & DSM 12768 & JCM 10985 \\
\hline Virgisporangium aurantiacum corrig. Tamura et al. 2001 & $\begin{array}{l}\text { IFO (now NBRC) } 16421= \\
\text { JCM } 11002\end{array}$ & CIP 107212 \\
\hline Virgisporangium ochraceum corrig. Tamura et al. 2001 & $\begin{array}{l}\text { IFO (now NBRC) } 16418= \\
\text { JCM } 11001\end{array}$ & CIP 107213 \\
\hline
\end{tabular}




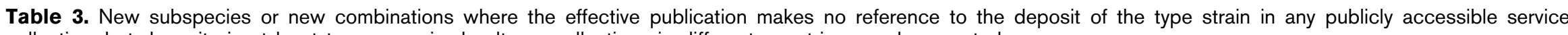
collection, but deposits in at least two recognized cultures collections in different countries are documented

\begin{tabular}{|c|c|}
\hline Name of taxon and author(s) & Accession number(s) under which type strain is available \\
\hline Achromobacter denitrificans (Rüger and Tan 1983) Coenye et al. 2003 & $\begin{array}{l}\text { ATCC } 15173=\text { CCUG } 407=\text { CIP } 77.15=\text { DSM } 30026=\text { IFO }(\text { now NBRC }) 15125=\mathrm{JCM} \\
5490=\text { LMG } 1231=\text { NCTC } 8582\end{array}$ \\
\hline Clostridium stercorarium subsp. leptospartum (Toda et al. 1989) Fardeau et al. 2001 & ATCC $51338=$ DSM $9219=$ IAM 13499 \\
\hline Clostridium stercorarium subsp. stercorarium Madden 1983 & ATCC $35414=$ DSM $8532=$ NCIB 11754 \\
\hline Clostridium stercorarium subsp. thermolacticum (Le Ruyet et al. 1988) Fardeau et al. 2001 & ATCC $43739=$ DSM 2910 \\
\hline Novosphingobium aromaticivorans corrig. (Balkwill et al. 1997) Takeuchi et al. 2001 & $\begin{array}{l}\text { ATCC } 700278=\text { CIP } 105152=\text { DSM } 12444=\text { HAMBI } 2257=\text { IFO (now NBRC) } 16084= \\
\text { SMCC F } 199\end{array}$ \\
\hline Novosphingobium capsulatum (Leifson 1962) Takeuchi et al. 2001 & $\begin{array}{l}\text { ATCC } 14666=\text { CCUG } 17697=\text { CCUG } 31202=\text { CIP } 82.103=\text { DSM } 30196=\text { GIFU } 11526= \\
\text { HAMBI } 103=\text { IFO }(\text { now NBRC) } 12533=\mathrm{JCM} 7508=\mathrm{JCM} 7452=\mathrm{LMG} 2830=\mathrm{VKM} \\
\text { B-1564 }\end{array}$ \\
\hline Novosphingobium rosa corrig. (Takeuchi et al. 1995) Takeuchi et al. 2001 & $\begin{array}{l}\text { ATCC } 51837=\text { DSM } 7285=\text { HAMBI } 2068=\text { IFO }(\text { now NBRC) } 15208=\text { LMG } 17328= \\
\text { NCPPB } 2661\end{array}$ \\
\hline Novosphingobium stygium corrig. (Balkwill et al. 1997) Takeuchi et al. 2001 & ATCC $700280=$ CIP $105154=$ DSM $12445=$ IFO $($ now NBRC) $16085=$ SMCC B0712 \\
\hline Novosphingobium subarcticum (Nohynek et al. 1996) Takeuchi et al. 2001 & CIP $105288=$ DSM $10700=$ HAMBI $2110=$ IFO $($ now NBRC) $16058=J C M 10398$ \\
\hline Novosphingobium subterraneum corrig. (Balkwill et al. 1997) Takeuchi et al. 2001 & ATCC $700279=$ CIP $105153=$ DSM $12447=$ IFO $($ now NBRC) $16086=$ SMCC B0478 \\
\hline Planomicrobium mcmeekinii (Junge et al. 1998) Yoon et al. 2001 & ATCC $700539=$ CIP 105673 \\
\hline Planomicrobium okeanokoites (ZoBell and Upham 1944) Yoon et al. 2001 & $\begin{array}{l}\text { ATCC } 33414=\mathrm{CCM} 320=\mathrm{CIP} 105082=\mathrm{IFO}(\text { now NBRC) } 12536=\mathrm{LMG} 4030=\mathrm{NCIMB} \\
561=\mathrm{VKM} \text { B-1175 }\end{array}$ \\
\hline Propionivibrio pelophilus (Meijer et al. 1999) Brune et al. 2002 & CIP $106101=$ DSM 12018 \\
\hline Sphingobium chlorophenolicum (Nohynek et al. 1996) Takeuchi et al. 2001 & $\begin{array}{l}\text { ATCC } 33790=\text { CIP } 104885=\text { DSM } 7098=\mathrm{IFO}(\text { now NBRC) } 16172=\mathrm{JCM} 10275=\mathrm{LMG} \\
17771\end{array}$ \\
\hline Sphingobium herbicidovorans (Zipper et al. 1997) Takeuchi et al. 2001 & CIP $106705=$ ATCC $700291=$ DSM $11019=$ IFO (now NBRC) $16415=$ LMG 18315 \\
\hline Sphingobium yanoikuyae (Yabuuchi et al. 1990) Takeuchi et al. 2001 & $\begin{array}{l}\text { ATCC } 51230=\text { CCUG } 28380=\text { CCUG } 31205=\text { CIP } 106726=\text { DSM } 7462=\text { GIFU } 9882= \\
\text { HAMBI } 1842=\text { IFO }(\text { now NBRC) } 15102=\text { JCM } 7371=\text { LMG } 11252\end{array}$ \\
\hline Sphingopyxis macrogoltabida (Takeuchi et al. 1993) Takeuchi et al. 2001 & $\begin{array}{l}\text { ATCC } 51380=\text { CIP } 104196=\text { DSM } 8826=\text { HAMBI } 1841=\text { IFO }(\text { now NBRC }) 15033=\text { LMG } \\
17324\end{array}$ \\
\hline Sphingopyxis terrae (Takeuchi et al. 1993) Takeuchi et al. 2001 & $\begin{array}{l}\text { ATCC } 51381=\text { CIP } 104198=\text { DSM } 8831=\text { HAMBI } 1834=\text { IFO }(\text { now NBRC }) 15098=J C M \\
10195=\text { LMG } 17326\end{array}$ \\
\hline Sporosarcina globispora (Larkin and Stokes 1967) Yoon et al. 2001 & $\begin{array}{l}\text { ATCC } 23301=\text { CCUG } 7419=\text { CIP } 103266=\text { DSM } 4=\text { HAMBI } 471=\text { IFO }(\text { now NBRC }) \\
16082=\text { JCM } 10046=\text { LMG } 6928=\text { NRRL NRS-1533=NRRL B-3396=VKM B-1435 }\end{array}$ \\
\hline Sporosarcina pasteurii (Miquel 1889) Yoon et al. 2001 & $\begin{array}{l}\text { ATCC } 11859=\text { CCUG } 7425=\text { CIP } 66.21=\text { DSM } 33=\text { LMG } 7130=\text { NCCB } 48021=\text { NCTC } \\
4822=\text { NRRL NRS- } 673=\text { VKM B-513 }\end{array}$ \\
\hline Sporosarcina psychrophila (Nakamura 1984) Yoon et al. 2001 & $\begin{array}{l}\text { ATCC } 23304=\text { CCM } 2117=\text { CCRC } 11738=\text { CCUG } 28886=\text { CIP } 103267=\text { DSM } 3=\text { IAM } \\
12468=\text { IFO (now NBRC) } 15381=J C M 9075=\text { LMG } 6929=\text { NRRL B-3397 = NRRL } \\
\text { NRS- } 1530\end{array}$ \\
\hline Zobellia uliginosa (ZoBell and Upham 1944) Barbeyron et al. 2001 & $\begin{array}{l}\text { ACAM } 538=\text { ATCC } 14397=\text { CCUG } 33448=\text { CECT } 4277=\text { CIP } 104808=\text { DSM } 2061=\text { IFO } \\
(\text { now NBRC) } 14962=\text { LMG } 3809=\text { NCIMB } 1863\end{array}$ \\
\hline
\end{tabular}


Table 4. New species where the protologue in the effective publication makes reference to the deposit of the type strain in either a single recognized culture collection or in two recognized culture collections in the same country, but another recognized culture collection in a different country is cited in the Abstract

\begin{tabular}{|c|c|c|}
\hline Name of taxon and author(s) & $\begin{array}{l}\text { Accession number(s) } \\
\text { cited in the protologue }\end{array}$ & $\begin{array}{l}\text { Accession number(s) } \\
\text { cited in the Abstract }\end{array}$ \\
\hline Arcanobacterium pluranimalium Lawson et al. 2001 & CCUG 42575 & CCUG $42575=$ CIP 106442 \\
\hline Corynebacterium casei Brennan et al. 2001 & LMG S-19264=DPC 5298 & LMG S-19264=NCIMB 30130 \\
\hline Corynebacterium mooreparkense Brennan et al. 2001 & LMG S-19265=DPC 5310 & LMG S-19265= NCIMB 30131 \\
\hline
\end{tabular}

This Request for an Opinion also documents problems which have arisen because insufficient attention has been paid to the workings and purpose of the Bacteriological Code (Lapage et al., 1992). While this may appear trivial, few microbiologists realize that, due to the efforts of a small group of dedicated systematists over the past 50 years, prokaryotic systematics has unique and comprehensive access to the information on names, the literature associated with them, type strains and the location of these strains, something which is the envy of those who wish to implement similar systems in botany and zoology. In paying attention to the workings and purpose of the Bacteriological Code (Lapage et al., 1992), it is hoped that this situation will be maintained, despite dwindling resources for systematics worldwide. Obviously, some of the problems listed here are due to suboptimal communication between the Judicial Commission, the ICSP, the Editors of the IJSEM, as well as the authors of papers submitted for valid publication of new names and new combinations. We hope that highlighting this problem will positively attract the attention of those involved and contribute to improving the current situation. As a result, prokaryotic systematics may maintain its role in leading the way in presenting accurate and comprehensive information to its end users.

Lists of abbreviations of collections and institutions cited in the tables are provided in several files available on the Internet. For example: 'LBSN - Culture collections of prokaryotes' (http://www.bacterio.cict.fr/collections.html); 'DSMZ - Abbreviations of Collections and Institutions' (http://www.dsmz.de/species/abbrev.htm); or 'WDCM Home Pages of Culture Collections in the World' (http:// wdcm.nig.ac.jp/hpcc.html).

References of original publications may be found in 'Bacterial Nomenclature Up To Date' (http://www.dsmz. de/bactnom/bactname.htm) or in the 'List of Bacterial Names with Standing in Nomenclature' (http://www. bacterio.cict.fr).

\section{References}

De Vos, P. \& Trüper, H. G. (2000). Judicial Commission of the International Committee on Systematic Bacteriology. IXth International (IUMS) Congress of Bacteriology and Applied Microbiology. Minutes of the meetings, 14, 15 and 18 August 1999, Sydney, Australia. Int J Syst Evol Microbiol 50, 2239-2244.

Labeda, D. P. (2000). International Committee on Systematic Bacteriology. IXth International (IUMS) Congress of Bacteriology and Applied Microbiology. Minutes of the meetings, 14 and 17 August 1999, Sydney, Australia. Int J Syst Evol Microbiol 50, 2245-2247.

Lapage, S. P., Sneath, P. H. A., Lessel, E. F., Skerman, V. B. D., Seeliger, H. P. R. \& Clark, W. A. (editors) (1992). International Code of Nomenclature of Bacteria (1990 Revision). Bacteriological Code. Washington, DC: American Society for Microbiology. 